\title{
Enhancing Business Intelligence by Means of Suggestive Reviews
}

\author{
Atika Qazi, ${ }^{1}$ Ram Gopal Raj, ${ }^{1}$ Muhammad Tahir, ${ }^{2,3}$ \\ Erik Cambria, ${ }^{4}$ and Karim Bux Shah Syed ${ }^{5}$ \\ ${ }^{1}$ Faculty of Computer Science and Information Technology, University of Malaya, Lembah Pantai, 50603 Kuala Lumpur, Malaysia \\ ${ }^{2}$ Faculty of Information Science and Technology, COMSATS Institute of Information Technology (CIIT), Park Road, \\ Islamabad 44000, Pakistan \\ ${ }^{3}$ Faculty of Computing and Information Technology, King Abdulaziz University, North Jeddah Branch, Jeddah 21589, Saudi Arabia \\ ${ }^{4}$ School of Computer Engineering, Nanyang Technological University, 50 Nanyang Avenue, Singapore 639798 \\ ${ }^{5}$ Faculty of Business and Accountancy, University of Malaya, Lembah Pantai, 50603 Kuala Lumpur, Malaysia
}

Correspondence should be addressed to Atika Qazi; atika@siswa.um.edu.my

Received 25 March 2014; Accepted 13 May 2014; Published 26 June 2014

Academic Editor: Jesualdo Tomás Fernandez-Breis

Copyright (C) 2014 Atika Qazi et al. This is an open access article distributed under the Creative Commons Attribution License, which permits unrestricted use, distribution, and reproduction in any medium, provided the original work is properly cited.

Appropriate identification and classification of online reviews to satisfy the needs of current and potential users pose a critical challenge for the business environment. This paper focuses on a specific kind of reviews: the suggestive type. Suggestions have a significant influence on both consumers' choices and designers' understanding and, hence, they are key for tasks such as brand positioning and social media marketing. The proposed approach consists of three main steps: (1) classify comparative and suggestive sentences; (2) categorize suggestive sentences into different types, either explicit or implicit locutions; (3) perform sentiment analysis on the classified reviews. A range of supervised machine learning approaches and feature sets are evaluated to tackle the problem of suggestive opinion mining. Experimental results for all three tasks are obtained on a dataset of mobile phone reviews and demonstrate that extending a bag-of-words representation with suggestive and comparative patterns is ideal for distinguishing suggestive sentences. In particular, it is observed that classifying suggestive sentences into implicit and explicit locutions works best when using a mixed sequential rule feature representation. Sentiment analysis achieves maximum performance when employing additional preprocessing in the form of negation handling and target masking, combined with sentiment lexicons.

\section{Introduction}

The emergence of Web 2.0 technologies and the growing number of online reviews websites, such as Amazon, Epinions, and Cnet, emphasize user participation. People are encouraged to express their opinions/sentiments on purchased products. Sentiment analysis (also commonly referred to as opinion mining) is a natural language processing task that aims to track the public's mood regarding a particular product or service. This type of text analysis belongs to the field of natural language processing, computational linguistics, and text mining [1]. It is cumbersome and there is high time overhead for a human reader to find appropriate resources, extract opinion sentences, read, and then summarize them to obtain useful information. Thus, automated opinion detection and summarization systems are still required. Existing opinion mining approaches can be grouped into four main categories: keyword spotting, lexical affinity, statistical methods, and concept-level analysis [2]. Keyword spotting classifies text by effect categories based on the presence of unambiguous effect words such as happy, sad, afraid, and bored. Lexical affinity assigns arbitrary words a probable "affinity" to particular emotions. Statistical methods learn effective information by counting word cooccurrence frequencies from large annotated corpora [3-5]. Conceptlevel analysis consists in biologically inspired approaches that exploit the conceptual and effective information associated with multiword expressions (rather than single words) to infer emotional or polarity values from natural language opinions [6-8].

All opinion mining approaches are performed on reviews, which can be (1) regular or (2) comparative. The review types 
are differentiated based on language constructs that express diverse kinds of information [9]. Regular opinions pertain to a single entity only, and comparative opinions juxtapose two or more entities [9]. The study of these two sorts of opinions motivates the classification of more useful review types, such as suggestions. Where the suggestions are recently introduced as a third type of review in the study of opinion mining examined by Qazi et al. in [10]. Extracting suggestive sentences from text is valuable for numerous applications in the business, medical, and e-learning environments, among others. Clearly, suggestions on products/features are not only useful for product manufacturers, but also useful to potential customers who can better utilize products by keeping in view suggestions to avoid problems and take advantage of optimal product benefits.

Suggestions are indirect speech acts. Kumar [11] explains that speech acts meant to direct someone to do something through suggestions are classified as suggestive. Suggestions, on the other hand, are either (1) suggestive with expressed locution or (2) suggestive with implied locution [12]. The expressed suggestive forms are further split into two types: (1) explicit performatives and (2) implicit performatives, where explicit performatives are sentences expressed with performative verbs and implicit performatives are phrases that use "modals" to express the statement [13-15]. In the second type, that is, the implied suggestive, it can be said that reason or precondition depends upon the reader's inference. For instance, a simple opinion sentence about a person may be " $\mathrm{Mr}$. $\mathrm{X}$ is very lazy." An explicit phrase could be "I suggest using blue for better results," while an implicit phrase may be "Let us go to the new café." Few phrases are interrogative and imperative, and an example of an interrogative suggestive may be "Why doesn't she/he/it.??" and an imperative suggestive may refer to the "let us" suggestive.

Generally, suggestive sentences employ quite different language constructs from typical opinion sentences. Hereby, the aim is to study the issue of identifying suggestive sentences in text documents, for example, consumer reviews of products like movies and cell phones. The issue is challenging because although it is obvious that the above example sentences all contain some suggestive indicators, their semantic is not as suggestive as, "I could go to buy this phone." Similarly, many sentences do not contain such indicators and are still suggestive sentences, like for instance "He might help you." We first classify suggestive sentences into different categories based on existing linguistic research and then expand them into additional categories that are essential in practice. Subsequently, we propose a novel approach based on mixed sequences and supervised learning to identify suggestive sentences. A sequence of items is denoted as $S=$ $\left\langle i_{1}, i_{2}, \ldots, i_{n}\right\rangle$, where every item corresponds to a feature that belongs to a certain token. The fundamental notion is a mixed sequence approach to achieve high recall and to build a supervised machine learning model to automatically classify each sentence into two classes: (1) suggestive and (2) nonsuggestive, in order to enhance precision. To sum up, the contributions made through this study are as follows: (1) propose a study of the problem with classifying suggestive sentences in text (to the best of our knowledge, there is no reported study on this matter so far); (2) categorize suggestive sentences into different types based on linguistic research; (3) perform sentiment analysis on classified suggestive reviews.

An effective approach to solve the drawback with feature construction using mixed sequences and the machine learning technology is thus proposed. This paper is organized as follows: in Section 2, related work is presented; Section 3 proposes the problem statement and categorizes different types of suggestive sentences, expanding on what is already available in linguistics; Section 4 presents the proposed technique; finally, Section 5 concludes the paper and suggests future directions.

\section{Related Work}

Related works from both computer science and linguistics are explored. Researchers in linguistics focus primarily on defining the syntax and semantics of suggestive constructs. Human communication is a broad phenomenon with multifarious facets and dimensions, including all signs whether textual, nontextual, verbal, and nonverbal. The majority of human communication occurs by means of utterances. According to linguists, suggestives are speech acts. The speech act theory was put forth by John Austin in his lectures published on how to do things with words [16]. Crystal [17] explained that according to Austin, there are two kinds of utterances, namely, constatives and performatives. Constatives are "statements that convey information" and performatives "do not communicate information, but are alike to actions," examples of which include "I name this ship..." and "I promise. .." [13].

Thus, language not only conveys information, but there are acts carried out or performed through words. Cook [18] elucidated that the speech act theory begins with the observation that there is a class of highly ritualistic utterances which carry no information about the world outside language at all, because they refer only to themselves. Examples of such declarations are swearing an oath, sentencing a criminal, opening a building, arresting a felon, or naming a ship. They are expressions in which saying the words and doing the action are the same thing: the function is created by the form. Searle [19] additionally contributed to this speech act theory. Crystal [17] further explained that there are direct speech acts and indirect speech acts, with suggestives falling in the indirect speech acts group. Indirect speech acts do not have an imperative form. Speech acts are observed to be addressing a listener directly, but most of them in mundane interactions are indirect $[13,17]$. One instance is the variety of modes of requesting that someone carry out an action. "Close the widow," for example, may carry a request in one situation and may convey a harsh tone to the listener.

Among the examples provided by Crystal to illustrate indirect speech acts are requests and suggestives. Consider the following example by Crystal: "It's getting cold in here, Shall we keep out the draught?" [17]. This speech act discussion given by Crystal shows that suggestives address the listener's "desire to perform the action or the speaker's reasons for having the action done." Semantic analysis relies on logic which cannot be applied as a method of discerning suggestive 
reviews. The focus of these research work is on a limited set of suggestive constructs containing keywords like we/I suggest/propose, why does not he/she/it, ought to, if I were you, what is the wrong with, suppose, etc. In brief, although linguists have studied suggestive phrases and grammar, they are more for human use than for automatic identification of suggestive sentences by computers.

In text and data mining, no direct work on suggestive sentences has been identified so far. Sentiment classification and opinion extraction are closely related but differ from our work. To classify stock postings, a manually crafted lexicon in conjunction with several scoring methods was employed by Das and Chen [20]. Several supervised machine learning methods for sentiment classification of movie reviews were examined by Pang et al. [3]. A number of learning methods for review classification were additionally experimented with, which show whether classifiers perform better than a sentence on a whole review [21]. A study by [22] investigated sentence subjectivity classification. A method of finding adjectives indicative of positive/negative opinions was discussed. A similar technique with respect to nouns was also discussed by [23]. Other related works on sentiment classification and opinion detection, emotion detection have been carried out [22, 24-31]. Different unsupervised and supervised techniques were proposed to analyze opinions in customer reviews as well [5, 32]. Liu's work was later progressed by $[33,34]$. Some literature is available on sentiment classification of comparative opinions. The difficulty with identifying comparative sentences and extracting comparative relations is addressed in some studies [9, 35-37]. From time to time, existing techniques are being explored and improved by researchers. However, none of these studies deal with suggestives, which is the focus of this work. To summarize, we are dealing with a new type of user input (suggestion) that is generally different from what is explored in existing studies. Such user input is valuable to users, potential buyers, and designers and is frequently available on ecommerce, blogs, and social media. Users are keen to share their experience with the public and also hope to learn from others' experience before they proceed with purchases. Hence, we argue the urgency of studying innovative types of reviews to aid product designers as well as customers in a wide range of helpful ways.

\section{Study Approach}

It is aimed via the work reported in this paper to fill a notable gap by offering designers and customers a possibility to process vast amounts of user input in the form of suggestive reviews. Initially, a linguistic view of suggestives is provided and some limitations are identified. They are then enhanced by classifying suggestive examples into suggestives with implicit and explicit locutions, as well as performing sentiment analysis on the suggestives.

3.1. Linguistic Perspective. As mentioned earlier in this discussion, suggestives are speech acts. More specifically, they are indirect speech acts. According to [11], speech acts suggesting someone to act in a particular way are known as suggestive. Most of suggestive sentence structures in English use the modal "should" which may imply a mild emphasis while communicating suggestion. Furthermore, the concept of suggestives becomes clearer once suggestives are compared with their contraries. Kumar maintained that directionals are the contraries of suggestives. The author explained that "the speech acts which are used to give direct instructions to someone to do something (as opposed to suggestives) are termed directional" [11]. Two broad types of suggestives have been identified: (1) expressed and (2) implied.

It is deduced that the opening keywords and phrases in suggestives are followed by infinitive verbs. Thus, constructions are should + infinitive, let us + infinitive, shall I + infinitive, do you want to + infinitive, why do not we/you + infinitive, we could/you could + infinitive, and ought to + infinitive. Clearly, most suggestive constructions follow a key suggestive word + infinitive verb structure. Nonetheless, there are exceptions, for example, I/we suggest/propose (suggest/propose that + subject + should + infinitive), if I were you, and what about/how about? (Key phrase (what about/how about) + gerund + noun). The above linguistic classification of suggestive sentences has two limitations: (1) nonsuggestive with suggestive words (in linguistics, sentences that contain suggestive indicators may not be used for identifying suggestive phrases, for example, in the phrase "In the context of cell phones, I did not suggest any one," there is no suggestion being offered here); (2) limited coverage or suggestives with nonsuggestive words (in practice, there are many suggestive sentences that do not contain any of the above suggestive words; this limitation comes under an implicit type of suggestives).

3.2. Proposed Enhancements. To address the first limitation, computational methods will be used (i.e., machine learning) to distinguish suggestive from nonsuggestive examples. As for the second drawback, several feature sets and the way they are written in combinations of other words potentially indicating suggestions will be employed. The performance characteristics of additional knowledge will be portrayed besides the bag-of-words representation and experimenting with a different, more flexible representation called Mixed Sequential Rules that is evaluated within a machine learning framework. We also test the effect of feature selection. For sentiment lexicons, the effects of target masking, negation handling, and lexicon features are investigated.

\section{Proposed Technique}

In the present work, suggestives are studied at the sentence level. Thus, the problem is stated in view of sentences. Task (1) given a review, $r$, predicts its class, $c$, where $c$ is a regular opinion, comparative or suggestive; task (2) given a suggestive review, $r$, predicts whether it is a suggestion with implicit or explicit locution; task (3) given a review, $r$, predicts whether its sentiment is a negative, neutral, or positive. As discussed earlier, a suggestive sentence is indicated both via specific keywords or more complex phrases and syntax patterns. 
Therefore, we have framed this problem as a supervised learning framework in an attempt to train a classification model capable of predicting whether a particular sentence contains a suggestion based on existing examples, which have already been classified as suggestions or not. In order to achieve superior classification performance and discover useful feature sets, we experiment with several combinations of feature sets and classification models. The feature sets are designed to accommodate existing state of the art in text classification, along with novel feature sets that utilize linguistic intuition behind suggestive statements. First, the various feature sets employed in this evaluation are described. However, suggestions may also exist without an explicit suggestion word. Similarly, nonsuggestive sentences can contain a suggestion word. In order to accommodate these nontypical cases, a machine learning approach that works on a wider feature space, looking beyond suggestive keywords, is applied. Such methods do not merely identify features that are indicative of a suggestive sentence, but also counter-indicative features, meaning they can indicate that a certain sentence is definitely not a suggestion.

4.1. Bag-of-Words Features. Since every statement in its raw form is represented as a sequence of words, it cannot be fed directly to the learning algorithms themselves, as most expect input in the form of real-valued feature vectors with a fixed size rather than raw text documents of variable length.

To address this, the following preprocessing approaches are used:

(i) tokenizing strings and giving an integer identifier for each possible token by using whitespaces and punctuation as token separators;

(ii) transforming the individual tokens for the purpose of data cleaning. In this step, all words are lowercased and stemming is applied via the Snowball stemmer [38];

(iii) masking the names of the review products, as well as the products they are being compared with. For this preprocessing technique the notation " $M s k$ " is used. Any mention of the target product is replaced with "[Target]," and every mention of another product is replaced with "[Other];"

(iv) negation processing, transforming every word $w$ in a window of length 2 after a negation word into a "not$w$ " token. With this technique, the notation "Neg" is used. Literature demonstrates that this particular preprocessing approach can improve sentiment analysis performance [39];

(v) filtering the tokens in order to remove words with low information value. All tokens which occur less than three times in the training set are filtered;

(vi) counting how many times word tokens occur in each document.

Features and samples are defined as follows.

(i) Each individual token occurrence frequency (normalized or not) is treated as a feature. (ii) The vector of all the token frequencies for a given document is considered a Multivariate sample.

Description of documents depends upon word used in it with little or no care of where the word is used in the document. This follows that a corpus of documents can be depicted through a matrix form, whereby matrix $X$ with a row for each document and column for each token (i.e., word) taking place in the corpus. The value of cell $x_{i j}$ represents the number of times the word $j$ appears in the example. In our evaluation, this feature set is denoted as "Bow" (bag-ofwords). Since this approach is widely used in text classification, this feature set is deemed to be a baseline. Within preprocessing, part-of-speech tagging is performed as well, which is employed in other feature sets. The Maxent tagger, trained on the Penn Treebank features, is utilized to produce the part-of-speech tags [40].

4.2. Surface Features. This feature set covers peculiarities that may appear in informal texts, such as social media or public review boards. These features focus mainly on the style of expression and less on the content itself. This feature set is included to determine whether it carries any interesting information for suggestion classification:

(i) number of fully capitalized words in review "This phone is AWESOME" generates a value of 1.0;

(ii) number of question words (how, why, what, who, when, and where) "Why and how is this broken?" generates a value of 2.0 ;

(iii) number of negation words (not, never, neither, nobody, no, none, nor, nothing, and nowhere) which are words that negate meaning and can often affect the interpretation of the whole sentence, as revealed in the task of sentiment detection [20];

(iv) number of contrast phrases (contrast, by the same token, conversely, instead, likewise, on one hand, on the other hand, on the contrary, rather, similarly, yet, but, however, still, nevertheless, and in contrast) which are words that signal the presence of a contrasting statement and often imply that the user is evaluating a certain object;

(v) number of question marks. In the evaluation, this feature set is denoted as "Surf."

4.3. Suggestive Clues. Given the tokenized content annotated with part-of-speech tags, features are generated based on the presence of patterns given the theory described in Section 3. The tokenized and stemmed text is traversed and combined with its part-of-speech tag sequence to distinguish whether a pattern matches. If so, that feature's value is set to 1.0. For instance, the reviews "This phone is bad, I should have bought something else" and "If I were you, I'd stay away" produce the features $\{$ "should": 1, "if_i_were_you": 1$\}$. We hypothesize that these patterns alone carry a large portion of information necessary for the successful detection of suggestions. This features set is denoted by "Sug." 
4.4. Comparison Clues. Comparator keywords were successfully combined with class sequential rules for the task of detective comparative sentences [41]. Since comparative sentences are a disjointed set from suggestive sentences, these features are also evaluated for suggestion extraction.

4.5. Performative Verbs. To take into account the background theory on how suggestions are presented, a lexicon of performative verbs is additionally included [21]. We hypothesize that including an indicator of a certain statement containing a performative verb may be indicative that the speaker is judging whether a product deserves a positive or negative suggestion. This feature set emits a single feature, with its value corresponding to how many performative verbs were detected in the example. This feature set is denoted as "Per."

4.6. Sentiment Lexicon Features. Only for the sentiment analysis task, external knowledge is integrated in the current model by means of a sentiment lexicon. In particular, we use SenticNet, a knowledge base that maps a sentiment value from -1.0 to 1.0 to a set of words or multiword expressions [42]. The following features are generated:

(i) sum of all positive sentiments of all words;

(ii) sum of all negative sentiments of all words;

(iii) total objective sentiments of all words (where objective $=1.0-($ positive + negative $))$ score;

(iv) ratio of total positive to negative scores for all words.

In addition to total sums, the same features for nouns, verbs, adjectives, and adverbs are also generated. Besides providing total sums, these features are also produced for nouns, verbs, adjectives, and adverbs separately. This feature is set as "Lex."

4.7. Mixed Sequential Rules. A sequence of items is represented as $S=\left\langle i_{1}, i_{2}, \ldots, i_{n}\right\rangle$, where every item corresponds to a representation of a certain token. Recent methods for comparison classification have successfully used sequential patterns of part-of-speech tags surrounding certain keywords [9]. The researchers proposed a sequence construction method, which takes all sequences within a 3-token radius from keywords and replaces all the words except the keyword with their respective part-of-speech tag. To better explain the phenomenon, a method is herewith proposed that generates several features from the same token sequence, depending on representation level.

A mixed sequential rule feature generator is defined by two parts: a word of interest and its surroundings. Initially all token sequences are taken with lengths up to $n$ that contain at least one word of interest. Then the feature is generated by combining the literal representation of the word of interest, and the surrounding tokens are represented by their respective part-of-speech tags. The following rules define various classes of words of interest for generating sequential rule features.

(i) Every verb is a word of interest. For instance ("buy," "this," "camera" $\rangle$ becomes "buy_DT_NP." (ii) Every adjective is a word of interest. For instance 〈"buy," "this," "good" "camera"〉 becomes "VB_DT_good_NP."

(iii) Every adverb is a word of interest.

(iv) Every suggestive keyword is a word of interest.

(v) Every comparative keyword is a word of interest.

Through this procedure, all subsequences of lengths up to 3 are generated. Each occurrence of a sequence rule in a given example generates a single feature corresponding to that sequence rule.

4.8. Feature Combination. In order to join the contribution of multiple feature sets, features are combined by performing a union of all features on every individual example. For instance, in a "Surf + Sug" feature construction setting for the example "This is an AWESOME phone, you should buy it!" the following would be generated: \{"should": 1.0 "fully_capitalized_words": 1.0, "exclamation": 1.0\}. In the end, every known feature is enumerated and assigned a dimension in the vector space model, so that every column corresponds to a feature and every row corresponds to a review. The dataset is therefore represented as a matrix $X$, with $n$ rows and $d$ dimensions, where " $n$ " is the number of examples and " $d$ " is the number of known features in that particular feature combination.

4.9. Feature Selection. So as to reduce dimensionality and noisy features, applying the Chi-squared feature selection criterion [43] and maintaining the top $20 \%$ of the features are experimented with. The Chi-square statistic measures the lack of independence between the feature, $f$, and the class, $c$, given the training data which could be used to estimate extremeness based on Chi-square distribution with one degree of freedom. This feature selection approach selects only the features which are least likely to be class independent.

4.10. Learning Models. Supervised learning consists of learning the link between two datasets: data $X$ and an external variable that we are trying to predict, usually called target or label and is denoted as $y$, a one-dimensional array of class values for the examples. Support vector machines using the linear kernel are employed for experimentation [44], as it has been shown to perform well in text classification tasks with many features. Logistic regression with $L_{1}$ regularization also partakes in testing [21], and this has been shown to promote sparsity in the model, resulting in simpler models. Trials were done with different regularization parameter (the $C$ parameter) values. For instance, $L R \_C 5$ means that $L R$ with $C=5.0$ is applied.

The entire pipeline is visualized in Figure 1. First, the various feature construction methods are run on the input dataset, following which the various feature sets are combined into a common feature space, and feature selection is performed. Finally, the obtained single pruned feature space is used with a supervised machine learning model. This pipeline serves for both training the classification model and executing the actual predictions. 


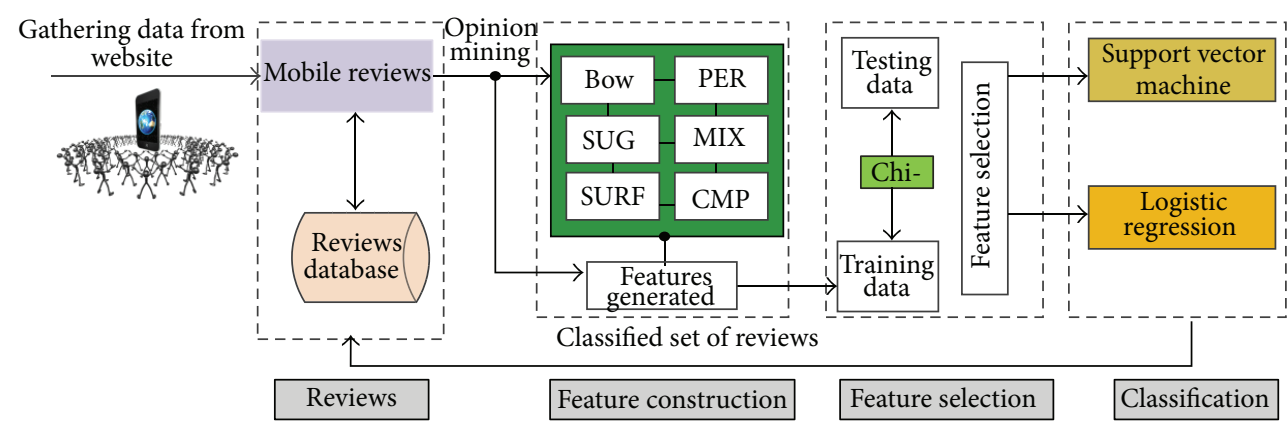

FIGURE 1: Suggestive and comparative detection workflow schematic.

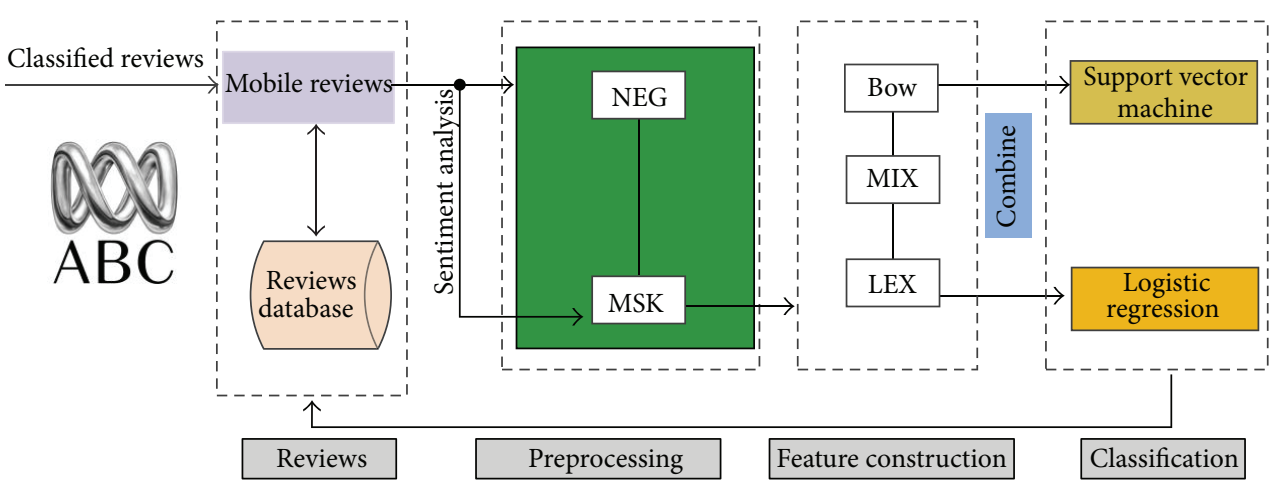

FIgURE 2: Sentiment analysis workflow schematic.

Figure 2 demonstrates a slightly different workflow pipeline. As some feature sets do not contribute significantly to performance, they are not present. The same applies to feature selection. On the other hand, the feature construction pipeline contains additional processing in the preprocessing step, namely, experimenting with the effect of negation handling and target masking.

4.11. Hypotheses. The baseline representation is a straightforward bag-of-words representation; one that we endeavor to improve. The hypotheses to be validated are:

(i) using suggestive key phrases improves performance;

(ii) using comparison key phrases improves performance;

(iii) using performative verb detection improves performance;

(iv) using the mixed sequential rules representation improves performance.

\section{Results and Discussion}

5.1. Experimental Setting. With the intention of discovering the key features, several different feature set combinations are evaluated. Besides, several supervised learning algorithms and their hyper parameters such as the logistic regression chi-square (LRChi $\left.{ }^{2}\right)$, logistic regression (LR), standard vector machine chi-square $\left(\mathrm{SVMChi}^{2}\right)$, and standard vector machine (SVM) are assessed. Each experiment is annotated by the feature sets and learning algorithm employed. The feature sets are marked with Bow, Cmp, Sug, Surf, and Mix. Where in case an experiment is marked with Bow + Cmp + Sug - SVM it means that its feature space is an amalgamation of bag-of-words features, comparison phrase indicator features and suggestive phrase indicator features, with SVM as the learning algorithm. These features are then transformed into a matrix representation and serve to train the learning model. The approach is evaluated on three classification setups:

(i) suggestive sentence detection: given an example, classify it as either suggestive or nonsuggestive;

(ii) comparative sentence detection: given an example, classify it as either comparative or noncomparative;

(iii) expressed/implied locutions detection: given an example suggestive sentence, classify it with either having expressed locution or implied locution.

The average, $F_{1}$, precision and recall obtained on 10fold cross-validation is reported. The dataset consists of 679 mobile phone reviews and is annotated with the type of review and its sentiment polarity. Out of the entire dataset, 181 are simple reviews, 189 are comparative reviews, and 309 are suggestive reviews. Of the suggestive reviews, 173 are suggestive with implicit locution, 100 with explicit locution, and 36 other suggestive reviews. The sentiment values were provided as star ratings ranging from 1 to 5 stars. One-star ratings were 


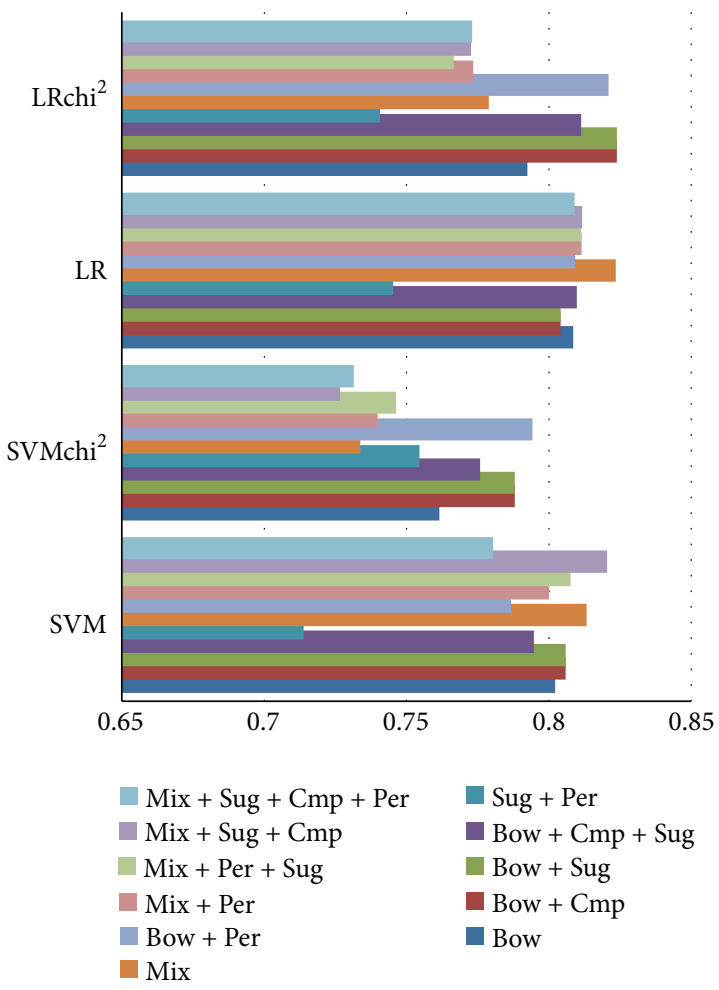

FIGURE 3: $F_{1}$ performance on suggestive classification.

treated as negative, 2-4-star reviews were considered neutral, and 5-star reviews were considered positive.

5.2. Suggestive Example Detection. Pertaining to suggestive sentence detection, Figure 3 shows a small but statistically significant improvement in/after combining a bag-of-words feature set with suggestive clues. Comparative clues and surface features do not seem to discernibly benefit this task at all. Due to the presence of bag-of-words features, which results in large feature dimensionality, feature selection by choosing the $20 \%$ most important features additionally enhances performance.

By looking deeper into the relationship between precision and recall displayed in Table 1 , it becomes obvious that while the bag-of-words baseline performs competitively, the combination of suggestive and comparison features offers a highprecision alternative with $91 \%$ precision.

5.3. Comparative Example Detection. Along the same lines, the issue of classifying comparative examples is also considered. It entails a disjointed set from the set of suggestive examples. The results have been obtained (see Figure 4 and Table 2).

Figure 4 and Table 2 demonstrate that a bag-of-words model augmented by incorporating comparative features works best for detecting comparative reviews. Similar performance levels are also attainable by employing a lexicon of performative verbs or a mixed sequence model. Furthermore,
TABLE 1: Performance numbers on $\mathrm{LRChi}^{2}$ over all feature construction approaches for detecting suggestive reviews.

\begin{tabular}{lccc}
\hline Feature construction & Precision & Recall & $F_{1}$ \\
\hline Bow & 0.8806 & 0.7603 & 0.8129 \\
Bow + Cmp & 0.8797 & 0.7539 & 0.8093 \\
Bow + Sug & 0.8715 & 0.7668 & 0.8124 \\
Bow + Cmp + Sug & 0.8796 & 0.7733 & 0.8193 \\
Bow + Cmp+ Sug + Surf & 0.859 & 0.7572 & 0.8008 \\
Sug & 0.899 & 0.6535 & 0.7534 \\
Mix & 0.8388 & 0.7671 & 0.7975 \\
Sug + Cmp & 0.9126 & 0.6537 & 0.7568 \\
Sug + Cmp + Surf & 0.8937 & 0.6699 & 0.7586 \\
Mix + Sug + Cmp & 0.8271 & 0.78 & 0.8005 \\
Mix + Surf + Sug + Cmp & 0.8309 & 0.7703 & 0.7972 \\
\hline
\end{tabular}

TABLE 2: Performance numbers on LR over all feature construction approaches for detecting comparative reviews.

\begin{tabular}{lccc}
\hline Feature construction & Precision & Recall & $F_{1}$ \\
\hline Bow & 0.7297 & 0.6658 & 0.6907 \\
Bow + Cmp & 0.8298 & 0.7013 & 0.7523 \\
Bow + Sug & 0.7983 & 0.65 & 0.7124 \\
Bow + Cmp + Sug & 0.7821 & 0.6961 & 0.731 \\
Sug + Per & 0 & 0 & 0 \\
Mix & 0.7611 & 0.7132 & 0.7302 \\
Bow + Per & 0.8175 & 0.6842 & 0.7409 \\
Mix + Per & 0.7861 & 0.6789 & 0.724 \\
Mix + Per + Sug & 0.7347 & 0.6947 & 0.7092 \\
Mix + Sug + Cmp & 0.7377 & 0.6816 & 0.7047 \\
Mix + Sug + Cmp + Per & 0.7551 & 0.6618 & 0.7003 \\
\hline
\end{tabular}

it has been shown that feature selection does not improve performance.

5.4. Suggestive Example Classification into Explicit and Implicit Locutions. Figure 5 and Table 3 provide the performance details of various approaches. Here, the blend of suggestive keywords and performative verbs offers the simplest, best approach for distinguishing between implicit and explicit locutions. The same performance level was also achieved by the mixed sequence rules, especially in combination with suggestive and comparison patterns. This demonstrates that even without domain knowledge, such as performative verb lexicons, suggestive patterns or comparative patterns, one may still be able to well-perform by training a on a dataset, preprocessed by a bag-of-mixed-sequences approach.

5.5. Sentiment Analysis. Figure 6 shows the performance details of sentiment analysis using various feature construction approaches. Here, the mixed sequences seem to make a good base feature set, capable of achieving adequate performance on its own and in some cases outperforming the bag-of-words representation. It is evident that the lexicons are insufficient when used alone, but they offer improved performance in combination with bag-of-words, masking, and 


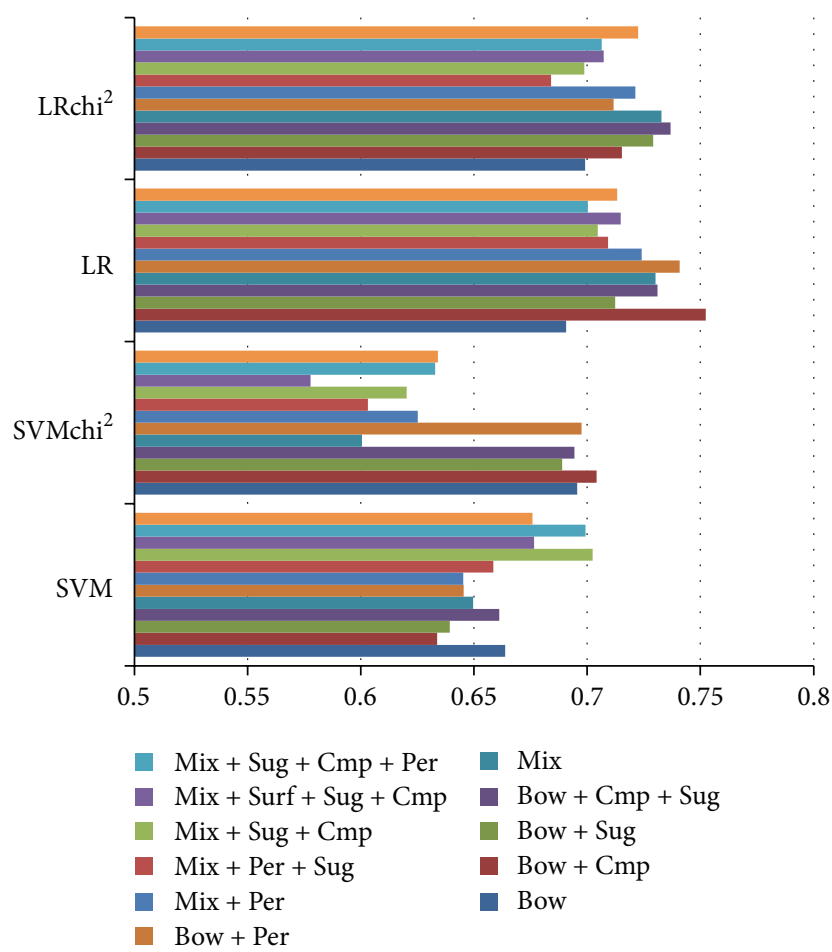

FIGURE 4: $F_{1}$ performance on comparative classification.

TABLE 3: Performance of $\mathrm{LRChi}^{2}$ on classification of explicit and implicit locution suggestions.

\begin{tabular}{lccc}
\hline Feature construction & Precision & Recall & $F_{1}$ \\
\hline Bow & 0.878 & 0.876 & 0.875 \\
Bow + Cmp & 0.881 & 0.904 & 0.890 \\
Bow + Sug & 0.862 & 0.918 & 0.887 \\
Bow + Cmp + Sug & 0.843 & 0.896 & 0.867 \\
Sug + Per & 0.822 & 0.938 & 0.872 \\
Mix & 0.909 & 0.925 & 0.915 \\
Bow + Per & 0.901 & 0.897 & 0.897 \\
Mix + Per & 0.902 & 0.914 & 0.906 \\
Mix + Per + Sug & 0.913 & 0.903 & 0.906 \\
Mix + Sug + Cmp & 0.909 & 0.931 & 0.918 \\
Mix + Sug + Cmp + Per & 0.906 & 0.921 & 0.911 \\
\hline
\end{tabular}

negation handling. The finest performance was obtained with the mixed sequential rule base feature set using negation handling and target masking in preprocessing, combined with SenticNet lexicon features.

Table 4 portrays the precision-recall trade-offs of the evaluated feature sets, indicating that while individual preprocessing mechanisms are insignificant on their own, they add up to some improvement.

5.6. Feature Analysis. Once a linear model has been trained with training data, its coefficients of individual features for every binary classifier are noted. For a given binary classifier, it is possible to observe whether an individual feature is indicative of the class or counterindicative-in which case its

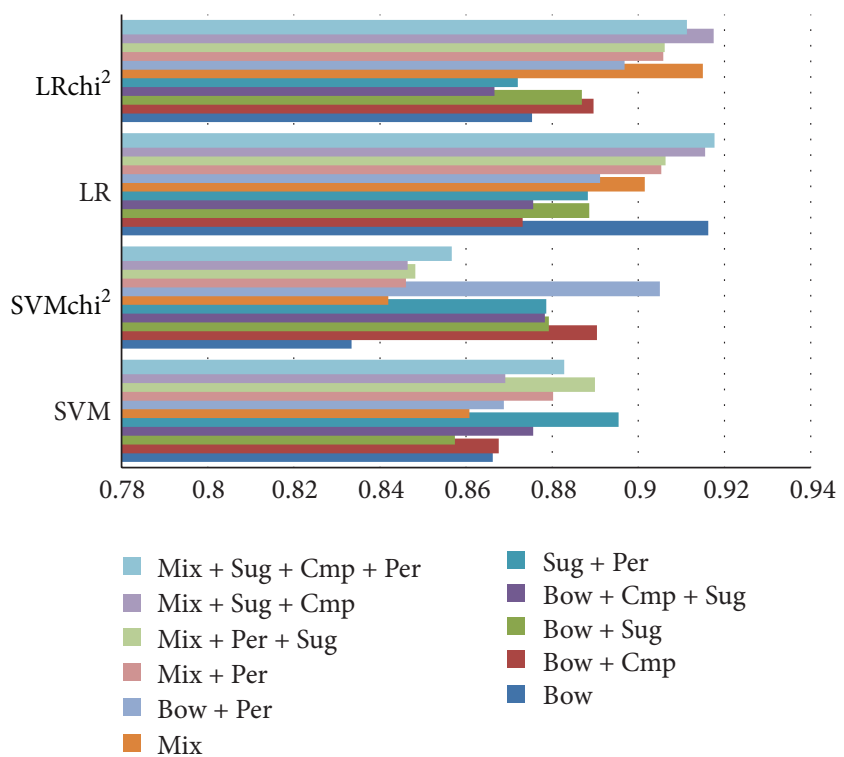

FIGURE 5: Performance numbers on sentiment analysis on suggestive sentences.

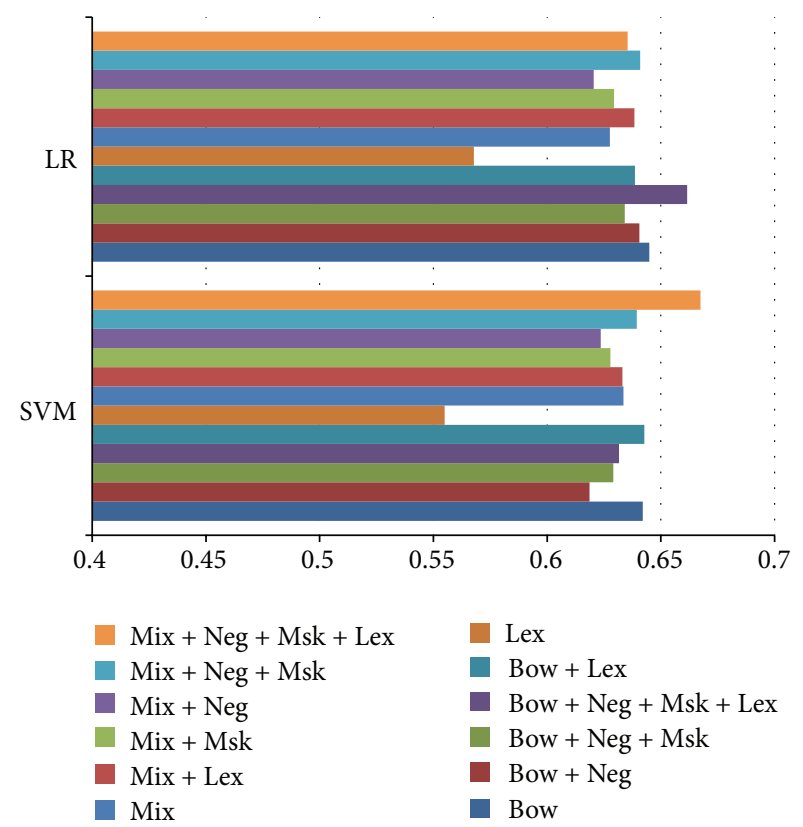

FIGURE 6: Performance numbers on various algorithms on separation of explicit and implicit locutions suggestions.

presence puts it in another class. For the task of classifying suggestive sentences in a bag-of-words model, the features with the lowest weights (signals for nonsuggestive sentences) were absolutely, company, better phone, prefer to, capture, functions, and ios, while the highest weights (indicative of suggestive sentences) were calendar, yet but, to text, fun, inches, not great, way of, let's, recently, and impressive. While these features make a well-performing classifier, they simultaneously remain very typical for the general topic of phone 
TABLE 4: Performance numbers on LR over all feature construction approaches for sentiment analysis of suggestive reviews.

\begin{tabular}{lccc}
\hline Feature construction & Precision & Recall & $F_{1}$ \\
\hline Bow & 0.636 & 0.679 & 0.645 \\
Bow + Neg & 0.632 & 0.657 & 0.641 \\
Bow + Neg + Msk & 0.628 & 0.648 & 0.634 \\
Bow + Neg + Msk + Lex & 0.655 & 0.693 & 0.662 \\
Bow + Lex & 0.628 & 0.658 & 0.639 \\
Lex & 0.581 & 0.665 & 0.568 \\
Mix & 0.629 & 0.678 & 0.628 \\
Mix + Lex & 0.632 & 0.669 & 0.638 \\
Mix + Msk & 0.616 & 0.662 & 0.629 \\
Mix + Neg & 0.608 & 0.652 & 0.621 \\
Mix + Neg + Msk & 0.634 & 0.666 & 0.641 \\
Mix + Neg + Msk + Lex & 0.626 & 0.672 & 0.635 \\
\hline
\end{tabular}

reviews; few of the features would successfully generalize to other domains.

As for the weights of a model trained only on suggestive clues, almost all of the clues have positive weights, whereas only "ought to" has a slight negative weight. This confirms that the theory holds true in this dataset but that some of the rules may benefit from restricting their context. For instance, "ought to" can also be used in a 3rd person scenario, where the reviewer is describing a hypothetical aspect of a device and is not actually giving a suggestion.

With regard to the weights of a suggestion detection model consisting of mixed sequence rules, when looking at the set of features that indicate nonsuggestions, patterns such as VB_good_., IN_more_than, charg_TO, CC_IN_was, should_be_ad, and realli_JJ are observed. These indicate qualitative device descriptors. Conversely, the suggestions are specified by features such as $P R P \_l o c k \_R B$, use_CC, will_need_TO,_go_RP, and catch_more_NN, patterns that, interestingly, indicate device usage. Following that, people who suggest a device also tend to more vividly describe themselves as using it. For the problem of characterizing suggestive sentences by classifying them into implicit or explicit locution, the model's behavior when using suggestive and comparative features is noted. Comparators indicate implicit locution, and suggestive clues tend to indicative explicit locution more. This confirms the theory that when a reviewer explicitly recommends a product, they are likely to use more explicit terms, such as modal verbs. Upon viewing the same task on a different feature set, the bestperforming mixed sequential rules, the pattern VBZ_not, $R B_{-}$worth $D T$, and TO_use_CD signifies implicit locution, while forget_TO_put and camera_is_RB are more indicative of explicit locution.

\section{Conclusion and Future Work}

A study of suggestive sentence classification and polarity detection was proposed in this paper. Different types of suggestive sentences were first analyzed from both linguistic and practical usage points of view. Aiming at identifying suggestive sentences, subsequent analysis was carried out through rule mining and machine learning approaches. The new approach was found effective by empirical evaluation based on mobile datasets.

Based on a comprehensive literature review and discussion, it can be argued that we spark a new debate on analyzing online product reviews. In order to construct models with outstanding performance in both suggestive and comparative tasks, the use of several feature sets was evaluated. Such feature sets may serve in detecting and characterizing suggestive sentences, and the combinations of various feature selection and supervised machine learning approaches were used. It was shown that for the task of detecting suggestive sentences, the basic bag-of-words model can be enhanced by augmenting it with additional features, especially with suggestive and comparative clues. The same approach also worked best in detecting comparative sentences. However, the bag-of-words representation may potentially lead to very topic-specific models, which may not translate to other domains, as indicated in the feature analysis. A combination of just suggestive and comparative keywords offers a highprecision alternative which can potentially also generalize better.

We would like to emphasize that, besides classifying suggestive reviews further in to explicit and implicit, we came across yet another novel type of reviews within this family. Although the finding of this type of review was not the main focus of this work, we have tentatively identified and reported it in this paper. Due to the lack of sufficient sample size (i.e., 36) we are cautious at this stage to classify these reviews separately within suggestive family. However, we do report this class for raising future research avenues based on larger sample size and deeper focused analysis on this type of reviews.

With respect to comparative sentence classification, no improvement was discerned when using a lexicon of performative verbs or a mixed sequential rule representation of the content. Nevertheless, it was demonstrated that in view of separating explicit and implicit locutions combining only the set of suggestive patterns with a lexicon of performative verbs offers optimal performance. Such performance level can be matched by training with mixed sequential rules. This indicates that flexible data representation is viable in instances where domain knowledge is unavailable. Since the dataset consists of a rather small number of examples, the hypothesis that feature selection is beneficial for both classification problems is confirmed, particularly when employing higher-dimensional representations based on the bag-ofwords model. With regard to sentiment analysis, the mixed sequence features proved to be a promising base feature set and sometimes outperform the bag-of-words feature set. Overall, the best strategy for this type of dataset was to employ negation handling and target masking on the input text and augment the bag-of-words feature set with additional lexicon features. From the perspective of learning algorithms, both logistic regression and support vector machines perform comparably well in the three issues discussed before.

Big social data analysis [45] plays a key role in business intelligence, as manufacturers need to be able to efficiently 
process user feedback in order to enhance decision making and create business policies best capable of launching new products. In particular, more focused reviews (e.g., suggestive) are better able to increase satisfaction levels, leading to successful sales. In summary, more helpful reviews are introduced, such as making suggestions by classifying such sentences in online reviews. This sort of user input is valuable to designers as well as users, and it is becoming gradually more available with the rise of e-commerce and new social media including blogs and social networks. It has been remarked that where sentiment analysis is still required, mostly opinion mining and sentiment analysis present domain-dependent concepts that render purely syntactical approaches ineffective. Therefore, there is great need for common-sense computing techniques [46] so that the cognitive and affective gap between word-level natural language data and concept-level opinions can be bridged. To this end, future work will apply the same review types for conceptlevel sentiment analysis in order to explore the generalization of suggestion detection and characterization on other domains.

\section{Conflict of Interests}

The authors declare that there is no conflict of interests regarding the publication of this paper.

\section{References}

[1] T. T. Thet, J.-C. Na, and C. S. G. Khoo, "Aspect-based sentiment analysis of movie reviews on discussion boards," Journal of Information Science, vol. 36, no. 6, pp. 823-848, 2010.

[2] E. Cambria and B. White, "Jumping NLP curves: a review of natural language processing research," IEEE Computational Intelligence Magazine, vol. 9, no. 2, pp. 48-57, 2014.

[3] B. Pang, L. Lee, and S. Vaithyanathan, "Thumbs up? Sentiment classification using machine learning techniques," in Proceedings of the Conference on Empirical Methods in Natural Language Processing (ACL '02), vol. 10, pp. 79-86, Association for Computational Linguistics, 2002.

[4] B. Pang and L. Lee, "Seeing stars: exploiting class relationships for sentiment categorization with respect to rating scales," in Proceedings of the 43rd Annual Meeting of the Association for Computational Linguistics (ACL '5), pp. 115-124, Association for Computational Linguistics, June 2005.

[5] M. Hu and B. Liu, "Mining and summarizing customer reviews," in Proceedings of the 10th ACM SIGKDD International Conference on Knowledge Discovery and Data Mining (KDD '04), pp. 168-177, ACM, August 2004.

[6] E. Cambria, A. Hussain, C. Havasi, and C. Eckl, "SenticSpace: visualizing opinions and sentiments in a multi-dimensional vector space," in Knowledge-Based and Intelligent Information and Engineering Systems, pp. 385-393, Springer, 2010.

[7] E. Cambria, A. Hussain, T. Durrani, C. Havasi, C. Eckl, and J. Munro, "Sentic computing for patient centered applications," in Proceedings of the IEEE 10th International Conference on Signal Processing (ICSP '10), pp. 1279-1282, 2010.

[8] E. Cambria, T. Mazzocco, A. Hussain, and C. Eckl, "Sentic medoids: organizing affective common sense knowledge in a multi-dimensional vector space," in Advances in Neural
Networks-ISNN 2011, vol. 6677 of Lecture Notes in Computer Science, pp. 601-610, 2011.

[9] N. Jindal and B. Liu, "Identifying comparative sentences in text documents," in Proceedings of the 29th Annual International ACM SIGIR Conference on Research and Development in Information Retrieval, pp. 244-251, ACM, August 2006.

[10] A. Qazi, R. G. Raj, M. Tahir, M. Waheed, S. U. Rehman, and A. Abraham, "A preliminary investigation of user perception and behavioural intention for different review types: customers and designers perspective," The Scientific World Journal, vol. 2014, Article ID 872929, 8 pages, 2014.

[11] R. Kumar, "Making machine translations polite: the problematic speech acts," Communications in Computer and Information Science, vol. 139, pp. 185-190, 2011.

[12] H. Sykes, "Pedagogies of censorship, injury, and masochism: teacher responses to homophobic speech in physical education," Journal of Curriculum Studies, vol. 36, no. 1, pp. 75-99, 2004.

[13] L. Fiorito, On Performatives in Legal Discourse, Metalogicon, 2006.

[14] J. Skelton, Language and Clinical Communication: This Bright Babylon, Radcliffe Publishing, 2008.

[15] W. Sinnott-Armstrong and R. J. Fogelin, Understanding Arguments: An Introduction to Informal Logic, Wadsworth Publishing Company, 2009.

[16] J. L. Austin, How to Do Things with Words, Oxford University Press, 1975.

[17] D. Crystal, Investigating English Style, vol. 47, Language, 1971.

[18] N. Cook, "Analysing performance and performing analysis," in Rethinking Music, pp. 239-261, 1999.

[19] J. R. Searle, "Speech acts and recent linguistics," Annals of the New York Academy of Sciences, vol. 263, pp. 27-38, 1975.

[20] S. R. Das and M. Y. Chen, "Yahoo! For Amazon: sentiment extraction from small talk on the Web," Management Science, vol. 53, no. 9, pp. 1375-1388, 2007.

[21] K. Dave, S. Lawrence, and D. M. Pennock, "Mining the peanut gallery: opinion extraction and semantic classification of product reviews," in Proceedings of the 12th International Conference on World Wide Web (WWW'03), pp. 519-528, ACM, May 2003.

[22] V. Hatzivassiloglou and J. M. Wiebe, "Effects of adjective orientation and gradability on sentence subjectivity," in Proceedings of the 18th Conference on Computational Linguistics, vol. 1, pp. 299-305, Association for Computational Linguistics, 2000.

[23] J. M. Wiebe, R. F. Bruce, and T. P. O’Hara, “Development and use of a gold-standard data set for subjectivity classifications," in Proceedings of the 37th Annual Meeting of the Association for Computational Linguistics on Computational Linguistics, pp. 246-253, Association for Computational Linguistics, 1999.

[24] N. Kobayashi, R. Iida, K. Inui, and Y. Matsumoto, "Opinion mining as extraction of attribute-value relations," in New Frontiers in Artificial Intelligence, pp. 470-481, Springer, 2006.

[25] L. W. . Ku, Y. T. Liang, and H. H. Chen, "Opinion extraction, summarization and tracking in news and blog corpora," in Pro-ceedings of the AAAI Spring Symposium on Computational Approaches to Analyzing Weblogs, 2006.

[26] T. Nasukawa and J. Yi, "Sentiment analysis: capturing favorability using natural language processing," in Proceedings of the 2nd International Conference on Knowledge capture, pp. 70-77, ACM, 2003.

[27] E. Riloff and J. Wiebe, "Learning extraction patterns for subjective expressions," in Proceedings of the Conference on 
Empirical Methods in Natural Language Processing, pp. 105-112, Association for Computational Linguistics, 2003.

[28] T. Wilson, J. Wiebe, and R. Hwa, "Just how mad are you? Finding strong and weak opinion clauses," in Proceedings pf the 19th National Conference on Artificial Intelligence (AAAI '04), pp. 761-769, July 2004.

[29] H. Yu and V. Hatzivassiloglou, "Towards answering opinion questions: separating facts from opinions and identifying the polarity of opinion sentences," in Proceedings of the Conference on Empirical Methods in Natural Language Processing, pp. 129136, Association for Computational Linguistics, 2003.

[30] C. Zhai, A. Velivelli, and B. Yu, "A cross-collection mixture model for comparative text mining," in Proceedings of the tenth ACM SIGKDD International Conference on Knowledge Discovery and Data Mining, pp. 743-748, ACM, 2004.

[31] N. Jatupaiboon, S. Pan-ngum, and P. Israsena, "Real-time EEGbased happiness detection system," The Scientific World Journal, vol. 2013, Article ID 618649, 12 pages, 2013.

[32] B. Liu, M. Hu, and J. Cheng, "Opinion observer: analyzing and comparing opinions on the web," in Proceedings of the 14th International Conference on World Wide Web, pp. 342-351, ACM, 2005.

[33] A.-M. Popescu and O. Etzioni, "Extracting product features and opinions from reviews," in Proceedings of the Conference on Human Language Technology and Empirical Methods in Natural Language Processing, pp. 339-346, Association for Computational Linguistics, October 2005.

[34] F. Moltmann, Coordination and Comparatives, Massachusetts Institute of Technology, 1992.

[35] N. Jindal and B. Liu, "Mining comparative sentences and relations," in Proceedings of the National Conference on Artificial Intelligence, p. 1331, AAAI Press, MIT Press, Menlo Park, Calif, USA, 2006.

[36] K. Xu, S. S. Liao, J. Li, and Y. Song, "Mining comparative opinions from customer reviews for Competitive Intelligence," Decision Support Systems, vol. 50, no. 4, pp. 743-754, 2011.

[37] K. Xu, W. Wang, J. S. J. Ren, J. Xu, L. Liu, and S. S. Y. Liao, "Classifying consumer comparison opinions to uncover product strengths and weaknesses," International Journal of Intelligent Information Technologies, vol. 7, no. 1, pp. 1-14, 2011.

[38] M. F. Porter, "Snowball: a language for stemming algorithms," 2001, http://snowball.tartarus.org/texts/introduction.html.

[39] M. Wiegand, A. Balahur, B. Roth, D. Klakow, and A. Montoyo, "A survey on the role of negation in sentiment analysis," in Proceedings of the Workshop on Negation and Speculation in Natural Language Processing, pp. 60-68, Association for Computational Linguistics, 2010.

[40] G. Malecha and I. Smith, "Maximum entropy part-of-speech tagging in NLTK," unpublished course-related report, 2010.

[41] Y. Yang and J. O. Pedersen, "A comparative study on feature selection in text categorization," in Proceedings of the 14th International Conference on Machine Learning, pp. 412-420, Morgan Kaufmann Publishers, 1997.

[42] E. Cambria, D. Olsher, and D. Rajagopal, "SenticNet 3: a common and common-sense knowledge base for cognition-driven sentiment analysis," in Proceedings of the AAAI Conference, Quebec City, Canada, 2014.

[43] P. D. Turney, "Thumbs up or thumbs down? Semantic orientation applied to unsupervised classification of reviews," in Proceedings of the 40th Annual Meeting On Association for Computational Linguistics (ACL '02), pp. 417-424, 2002.
[44] B. Pang, L. Lee, and S. Vaithyanathan, "Thumbs up? Sentiment classification using machine learning techniques," in Proceedings of the Conference on Empirical Methods in Natural Language Processing (EMNLP '02), pp. 79-86, Association for Computational Linguistics, 2002.

[45] E. Cambria, D. Rajagopal, D. Olsher, and D. Das, "Big social data analysis," in Big Data Computing, chapter 13, pp. 401-414, Taylor \& Francis, New York, NY, USA, 2013.

[46] E. Cambria, A. Hussain, C. Havasi, and C. Eckl, "Common sense computing: from the society of mind to digital intuition and beyond," in Biometric ID Management and Multimodal Communication, vol. 5707 of Lecture Notes in Computer Science, pp. 252-259, 2009. 

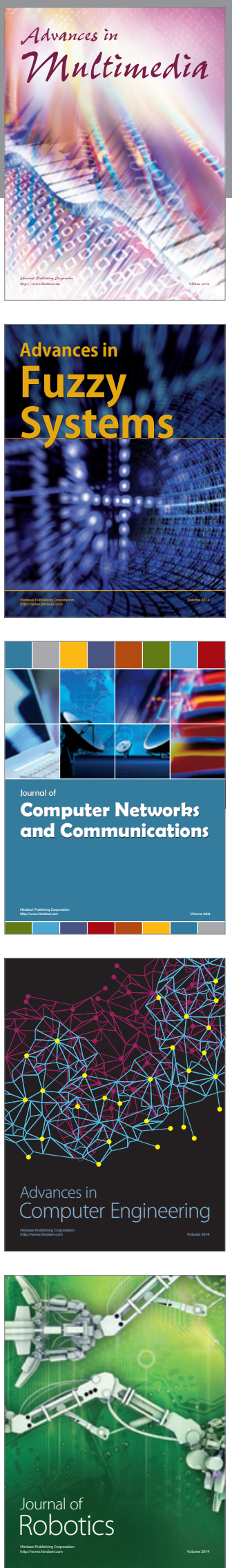

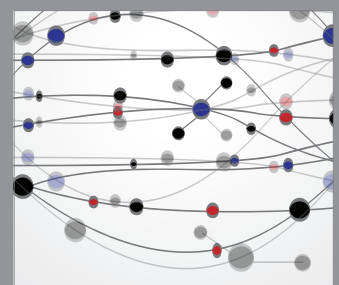

The Scientific World Journal
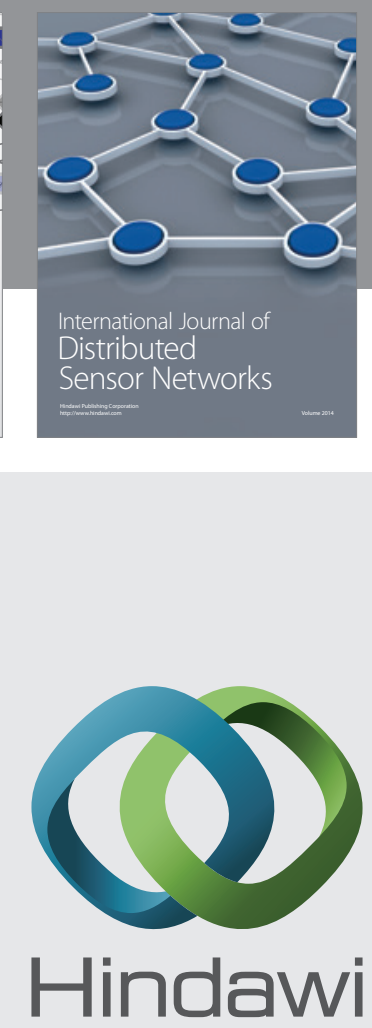

Submit your manuscripts at

http://www.hindawi.com
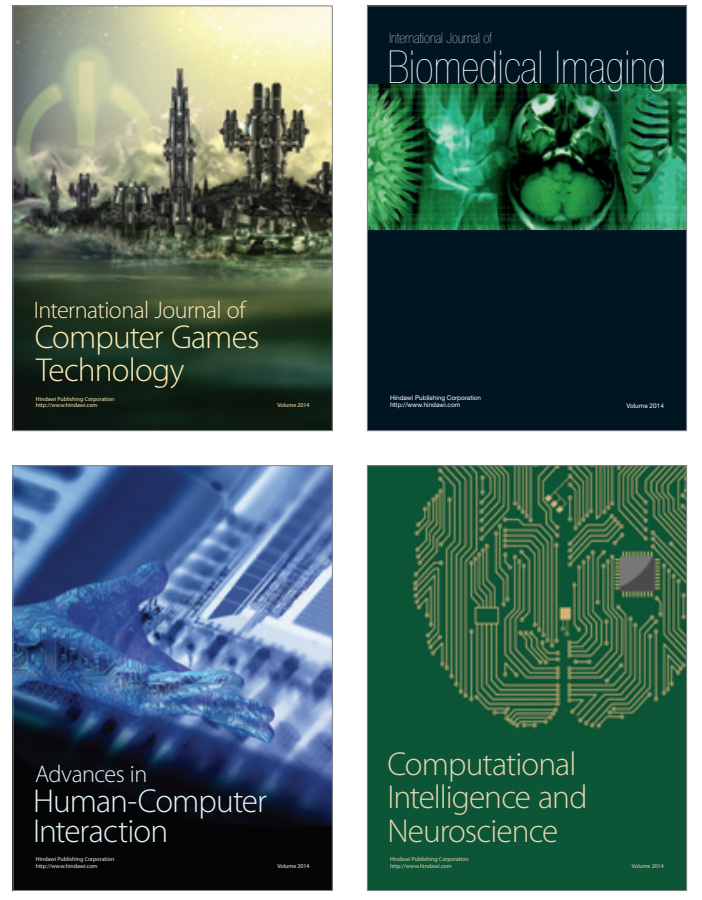
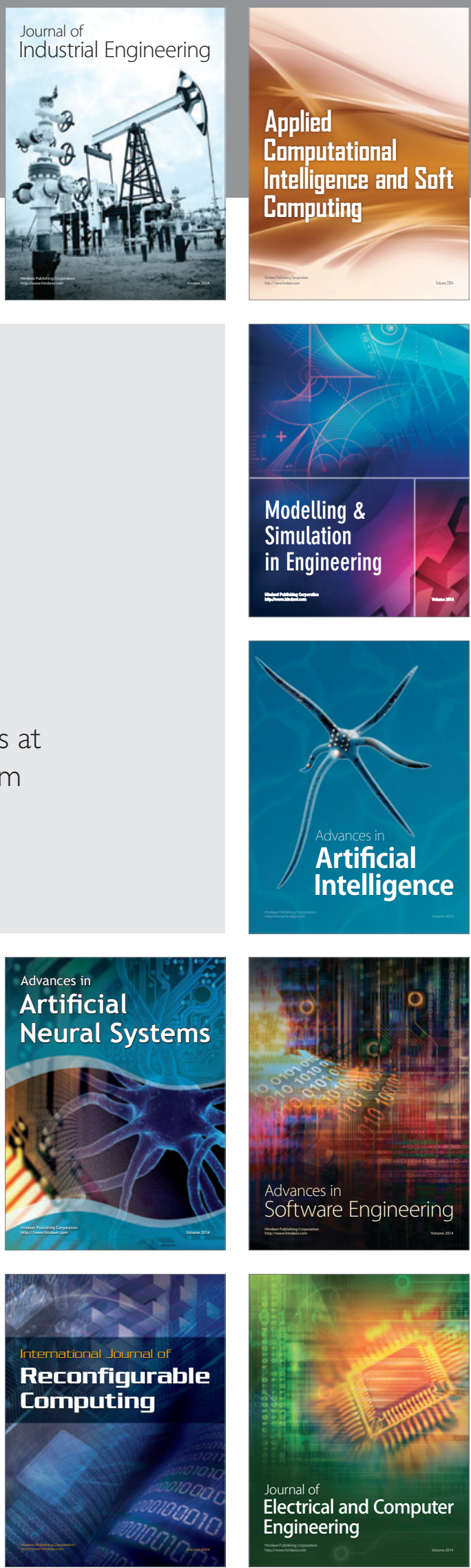